\title{
Models of Quantitative Variation of Flux in Metabolic Pathways
}

\author{
Peter D. Keightley \\ Department of Genetics, University of Edinburgh, Edinburgh EH9 3JN, Scotland \\ Manuscript received August 29, 1988 \\ Accepted for publication December 15, 1988
}

\begin{abstract}
As a model of variation in a quantitative character, enzyme activity variation segregating in a population is assumed to affect the flux in simple metabolic pathways. The genetic variation of flux is partitioned into additive and nonadditive components. An interaction component of flux variance is present because the effect of an allelic substitution is modified by other substitutions which change the concentrations of shared metabolites. In a haploid population, the proportion of interaction variance is a function of the gene frequencies at the loci contributing to the flux variation, enzyme activities of mutant and wild type at variable loci and activities at nonvariable loci. The proportion of interaction variance is inversely related to the ratio of mutant to wild-type activities at the loci controlling the enzyme activities. The interaction component as a function of gene frequencies is at a maximum with high mutant allele frequencies. In contrast, the dominance component which would apply to a diploid population is maximal as a proportion of the total when mutant alleles are at low frequencies. Unless there are many loci with large differences in activity between the alleles, the interaction component is a small proportion of the total variance. Data on enzyme activity variation from natural and artificial populations suggest that such variation generates little nonadditive variance despite the highly interactive nature of the underlying biochemical system.
\end{abstract}

$\mathrm{T}$ HE genetic variation of any character must be the result of genetic variation affecting basic biochemical processes. To what extent can advances in understanding biochemical systems improve our knowledge of the genetics of quantitative variation? Enzymology and studies of intermediary metabolism have provided insights into the actions of individual enzymes and the coupling of reactions in complex pathways represented graphically as the "metabolic map." A different approach, metabolic control theory (see review by KACSER and PorTeOus 1987), examines the effects of changes in enzyme activity on metabolic fluxes and metabolite concentrations. An analogous, but more complex, theoretical treatment of control of metabolic systems has been developed by SAVAGEAU (1969) and collaborators. [See Savageau, VoIT and IRVINE (1987) for a discussion of the relationship between the approaches.] Fluxes and metabolite concentrations are known as variables in the system while enzyme activities are under genetic control and are known as parameters. In metabolic control theory, the importance of an enzyme in controlling a flux is quantified by a "control coefficient" (KACSER and BURNS 1973), the fractional change in flux resulting from a small fractional change in enzyme activity. The effects of discrete changes in enzyme activity are important, however, because mutants can cause large changes. The problem of the general recessivity of "null" mutants at enzyme loci has been investigated by KACSER and BURNS (1981) who offered a general explanation based on the properties of metabolic pathways.
The effects of enzyme activity variation can be viewed at several levels. First, there is experimental evidence that enzyme activity itself is polygenically determined (e.g., PAIGen 1979; LAURIE-AhLBERG $e t$ al. 1980). At the next level are metabolic fluxes and metabolite pool concentrations. These are functions of perhaps all the enzymes in the "metabolic map," but are presumably more closely related to those enzymes in the pathway which "carries" the flux. At the third level are characters which are traditionally the subject of quantitative genetics, some of which have economic importance (e.g., growth rate, fatness), and likely to be influenced by many metabolic fluxes. Here, quantitative variation in metabolic flux is modeled by assuming that the phenotype (flux) is affected by enzyme activity variation segregating in a population. The flux is assumed to be a function of enzyme activity according to a simple biochemical model. The variance in flux is partitioned into additive and nonadditive components. This approach differs from classical models in quantitative genetics where the phenotypic variation usually depends on arbitrary assumptions of the effects of alleles. Here, the variation in the phenotype resulting from genetic variation depends on biochemical assumptions. It is known that because enzymes share common substrates, allelic substitutions at one locus modify the allelic effect at another (e.g., Dykhuizen, Dean and Hartl 1987), so epistatic interactions are automatically built into metabolic systems. The conditions necessary for substantial interaction variance in flux as a proportion of 
FiguRE 1.-The linear pathway. A chain of $n$ monomolecular unsaturated enzymes catalyses the conversion of externally controlled substrate $X_{A}$ to product $X_{B}$ via the intermediates $S_{1}$ to $S_{n-1}$.

the total genetic variance are investigated (the terms interaction and epistasis are used synonymously here). An attempt is made to relate experimentally determined variations in enzyme activities to the predictions of the models. Most of the formulations and simulations are restricted to the case of haploid populations. The consequences of diploidy and a dominance component of variance are discussed, however, in relation to results of previous investigations (KACSER and BURNS 1981; KEIGHTLEY and KACSER 1987).

\section{MODEL}

\section{Biochemical model}

Consider the following monomolecular transformation within a pathway of substrate $S_{i}$ to product $S_{j}$ catalyzed by enzyme $E_{\mathrm{j}}$.

$$
\ldots \rightleftharpoons \mathrm{S}_{\mathrm{i}} \underset{\mathrm{E}_{\mathrm{j}}}{\rightleftharpoons} \mathrm{S}_{\mathrm{j}} \rightleftharpoons \ldots
$$

The rate, $v_{j}$ of the reaction is given by

$$
v_{j}=\left(V_{j} / M_{i}\right)\left(S_{i}-S_{j} / K_{i, j}\right) /\left(1+S_{i} / M_{i}+S_{j} / M_{j}\right)
$$

(Cleland 1963), where $V_{j}$ is the maximal velocity ( $\left.V_{\mathrm{MAX}}\right), M_{i}$ is the Michaelis constant $\left(K_{\mathrm{m}}\right)$ of $\mathbf{E}_{\mathrm{j}}$ with respect to $S_{i}$, similarly $M_{j}$ is the Michaelis constant for $\mathrm{E}_{\mathrm{j}}$ with respect to $S_{\mathrm{j}}, S_{i}$ and $S_{j}$ are the concentrations of $\mathrm{S}_{\mathrm{i}}$ and $\mathrm{S}_{\mathrm{j}}$, respectively, and $K_{i, j}$ is the equilibrium constant for the step. Assume that the enzyme is acting in conditions of low saturation, that is, the Michaelis constants for the forward and backward reactions are substantially greater than $S_{i}$ and $S_{j}$, respectively. An approximate expression for the rate is therefore

$$
v_{j}=\left(V_{j} / M_{i}\right)\left(S_{i}-S_{j} / K_{i, j}\right) .
$$

The quantitative variation in the character metabolic flux is investigated in two types of pathway containing a number of such enzymes:

Linear pathway (Figure 1): The external substances $\mathrm{X}_{\mathrm{A}}$ (input) and $\mathrm{X}_{\mathrm{B}}$ (output) are assumed to be at constant concentrations determined by the external environment. This would be the case, for example, if $X_{A}$ were a nutrient supplied to the organism at a constant rate, similarly if $X_{B}$ were a waste or end product, changes in whose concentration due to the activity of the pathway can be ignored.

The system will reach a steady state at which all substrate concentrations are constant (the steady state concentrations) and the rates of all the steps in the pathway are equal to one another and hence to the pathway flux, $J$, the rate of consumption of $\mathrm{X}_{\mathrm{A}}$ and production of $X_{B}$. Expressions of type (2) may be

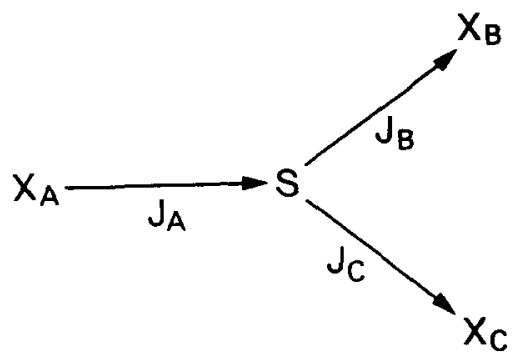

Figure 2.-The branched pathway. This consists of three linear branches as described in Figure 1 except that $S$ is the product of the common branch " $\mathrm{A}$ " and is the substrate of the reference " $\mathrm{B}$ " and competing " $\mathrm{C}$ " branches.
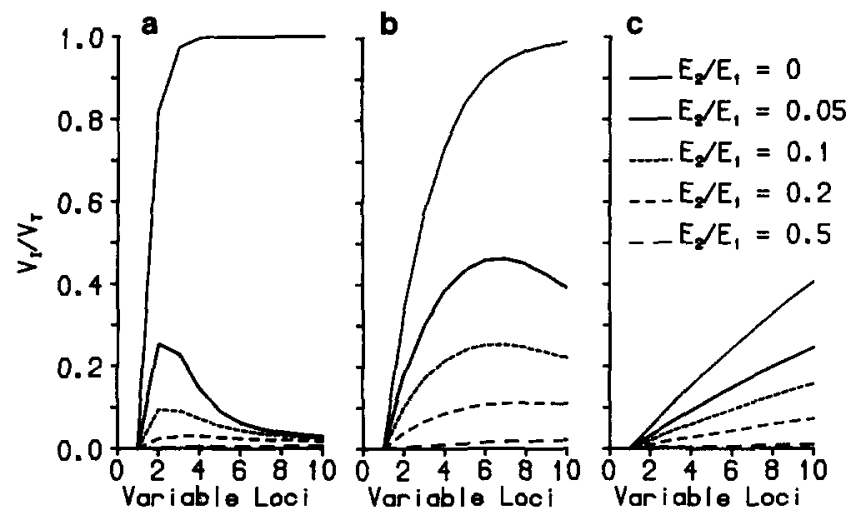

FIGURE 3.- The proportion of interaction variance of flux in a fixed length pathway of 10 equivalent loci. Each step on the horizontal axis corresponds to the introduction of one further variable locus into the population. The curves relate various values of ratio of mutant to wild-type activity, $E_{2} / E_{1}$. (a) $q=0.1$; (b) $q=0.5$; (c) $q$ $=0.9$.

written down for each step in the pathway which are a consistent set of simultaneous equations. Elimination of the variable $S$ terms gives an expression for the flux in terms of parameters only,

$$
J=\left(X_{\mathrm{A}}-X_{\mathrm{B}} / K_{\mathrm{A}, \mathrm{B}}\right) /\left(\sum_{i=1}^{n} M_{\mathrm{i}-1} / V_{i} K_{\mathrm{A}, \mathrm{i}-1}\right)
$$

(KACSER and BURNS 1973), where $X_{\mathrm{A}}$ and $X_{\mathrm{B}}$ are the concentrations of $\mathrm{X}_{\mathrm{A}}$ and $\mathrm{X}_{\mathrm{B}}$ respectively, $K_{\mathrm{A}, \mathrm{B}}=K_{\mathrm{A}, 1}$. $K_{1,2} \ldots K_{n-1, \mathrm{~B}}$ is the equilibrium constant between $\mathrm{X}_{\mathrm{A}}$ and $\mathrm{X}_{\mathrm{B}}$, similarly $K_{\mathrm{A}, i-1}$ is the equilibrium constant between $X_{A}$ and $S_{i-1}$, the first term in the summation containing $K_{\mathrm{A}, 0}=1, M_{0}$ is the Michaelis constant of $\mathrm{E}_{1}$ with respect to $\mathrm{X}_{\mathrm{A}}$, and $n$ is the number of steps in the pathway. Equation 3 may be rewritten as

$$
J=\left(X_{\mathrm{A}}-X_{\mathrm{B}} / K_{\mathrm{A}, \mathrm{B}}\right) /\left(\sum_{i=1}^{n} 1 / E_{i}\right) .
$$

The $E$ terms are proportional to the enzyme activities which are modifiable by mutations, and are also functions of equilibrium constants.

Branched pathway (Figure 2): As in the linear pathway, the concentrations of the external substances, in this case $\mathrm{X}_{\mathrm{A}}, \mathrm{X}_{\mathrm{B}}$, and $\mathrm{X}_{\mathrm{C}}$, are assumed to be constant and determined by the environment. 

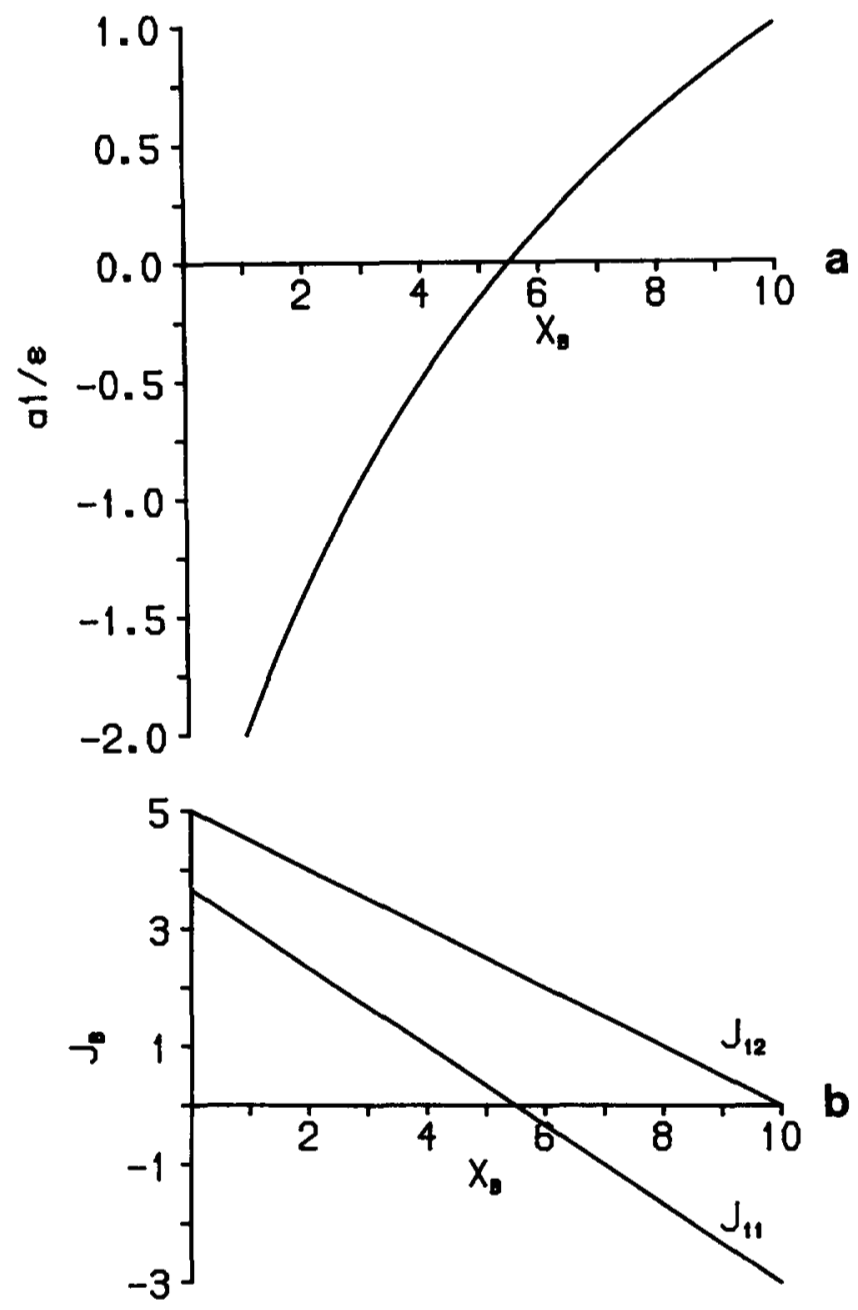

FIGURE 4.-The effect of asymmetry in the branched system. There are three enzymes in the system, one in each branch, and the wild-type activity of each is one. In the " $B$ " and " $C$ " branches a mutant allele of zero activity segregates in the population at frequency $1 / 2$. The value of $X_{A}$ is 10 and $X_{C}$ is 1 . (a), The effect on the value of $a_{1} / \epsilon=\left(J_{11}-J_{21}\right) /\left(J_{11}-J_{21}-J_{12}+J_{22}\right)$ of modulating $X_{B}$. (b), The effect on the value of the reference flux. $J_{11}$ is the value of the double wild-type flux and $J_{12}$ is wild type for the " $B$ " enzyme and mutant for the "C" enzyme.

There are three branches, and the following terminology is used to describe them: (1) the common branch is "A," (2) the reference branch is "B," and (3) the competing branch is " $\mathrm{C}$." At steady state, the flux in the common branch is the sum of the fluxes in the reference and competing branches, i.e.,

$$
J_{\mathrm{A}}=J_{\mathrm{B}}+J_{\mathrm{C}} \text {. }
$$

The substrate $S$ is catalyzed by the first enzyme in both the reference and competing branches which therefore compete for S. At steady state the rates of each step in the system can be described by equations of type (4) which equate to a branch flux. Together with relation (5), elimination of the variable $S$ from these equations yields an expression for the flux in the reference branch:

$$
\begin{aligned}
J_{\mathrm{B}}=\left[c\left(X_{\mathrm{A}}-X_{\mathrm{B}} / K_{\mathrm{A}, \mathrm{B}}\right)+a\left(X_{\mathrm{C}} / K_{\mathrm{S}, \mathrm{C}}\right.\right. \\
\left.\left.-X_{\mathrm{B}} / K_{\mathrm{S}, \mathrm{B}}\right)\right] /\left(a b+a c+b c / K_{\mathrm{A}, \mathrm{S}}\right)
\end{aligned}
$$

where the terms $a, b$, and $c$ are "group enzyme activities" (KeIGHTLEY and KACSER 1987), and $K_{\mathrm{A}, \mathrm{B}}, K_{\mathrm{S}, \mathrm{B}}$, $K_{\mathrm{S}, \mathrm{C}}$ and $K_{\mathrm{A}, \mathrm{S}}$ are equilibrium constants between $\mathrm{X}_{\mathrm{A}}$ and $X_{B}, S$ and $X_{B}, S$ and $X_{C}$, and $X_{A}$ and $S$, respectively. For example, the $a$ group enzyme activity is given by

$$
a=\sum_{i=1}^{n} 1 / E_{i}^{\mathrm{A}},
$$

where $n$ is the number of enzymes in the "A" (common) branch, and the $E$ terms are proportional to enzyme activity and have similar meanings as in (4). The $b$ and $c$ group activities are given by $b=\sum 1 / E_{i}^{\mathrm{B}}$ and $c=\Sigma 1 / E_{i}^{\mathrm{C}}$.

\section{Genetic model}

Basic assumptions: It is assumed that genetic variation affects enzyme activities in a population of haploid organisms. Each activity is assumed to be affected by variation at one locus only and there are up to two alleles at each locus. The allele of higher activity at locus $j$ is termed the wild type, $E_{1 j}$, and the lower activity allele is termed the mutant, $E_{2 j}$. The frequency of the wild-type allele at the locus is $q_{j}$. Linkage disequilibrium is assumed to be absent.

Variance components of flux: Let $n$ be the number of loci affecting the flux and $g$ be the number of different genotypes, which in a haploid population is $2^{n}$. Let $J_{i}$ be the value (i.e., flux) of genotype $i$, and $f_{i}$ be its frequency in the population. The total genetic variance is

$$
V_{\mathrm{T}}=\sum_{i=1}^{g}\left(J_{i}-m\right)^{2} f_{i},
$$

where $m=\sum J_{i} f_{i}$ is the population mean. The additive variance in flux is

$$
V_{\mathrm{A}}=\sum_{j=1}^{n} \alpha_{j}^{2} q_{j}\left(1-q_{j}\right),
$$

where $\alpha_{j}$ is the average effect of a gene substitution (FALCONER $1981 \mathrm{ch} .7$ ), which is the mean change in value resulting from the change of a mutant allele to a wild-type allele, and can be obtained by differentiating the mean flux with respect to gene frequency, $q_{j}$ (KoJIMA 1959). The resulting expression for $\alpha_{j}$ is,

$$
\alpha_{j}=\partial m / \partial q_{j}=\sum_{i=1}^{g} f_{i} J_{i}\left(\delta_{i j} / q_{j}-\left(1-\delta_{i j}\right) /\left(1-q_{j}\right)\right),
$$

where $\delta_{i j}$ is one if the genotype is wild type with respect to locus $j$, or zero if mutant. Substitution of an expression for flux [e.g., (4) or (6)] gives an equation for the variance in terms of biochemical parameters (e.g., 

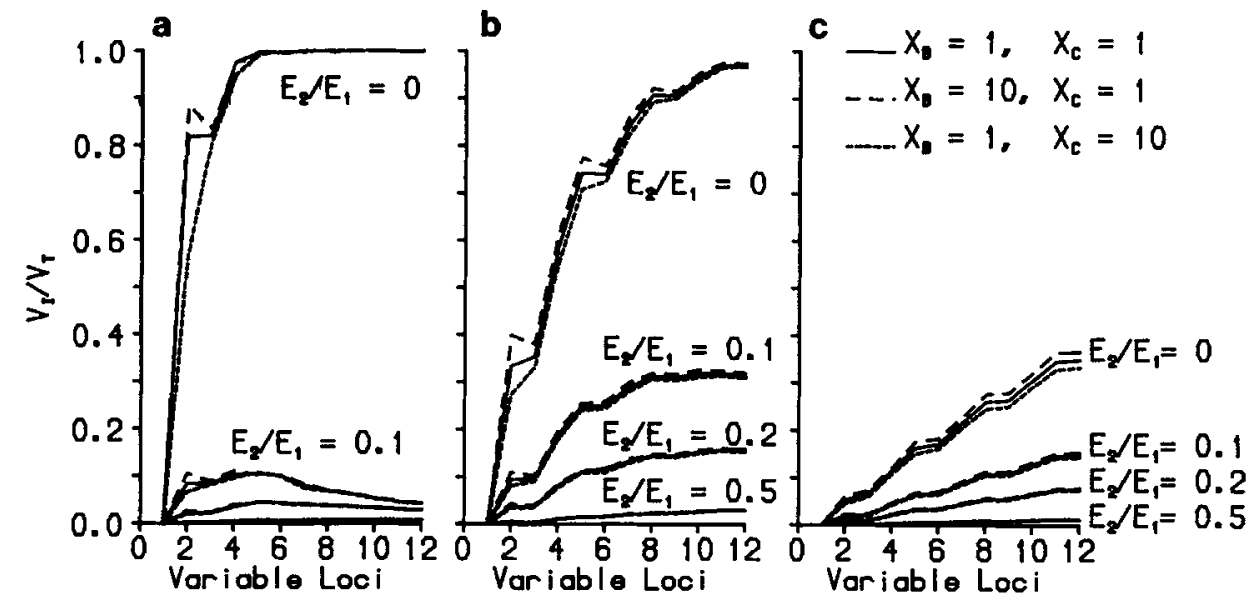

Figure 5.-The proportion of interaction variance in the reference flux of the branched pathway with variation simultaneously in three branches. Each branch has four equivalent enzymes. Each step on the horizontal axis corresponds to the introduction of one further variable locus into one branch and this occurs in the order A, B, C, $A$, and so on. The graphs relate various values of mutant to wild type activity ratio, $E_{2} / E_{1}$, and three types of system with varying degrees of asymmetry with respect to their thermodynamic properties. (a) $q=0.1$; (b) $q=0.5$; (c) $q=0.9$. enzyme activities). The interaction component is simply

$$
V_{\mathrm{I}}=V_{\mathrm{T}}-V_{\mathrm{A}}
$$

\section{RESULTS}

\section{Interaction in the linear chain}

Two-locus interactions: Assume that allelic variation in enzyme activity occurs at loci $j$ and $k$. With two variable loci there are therefore four haploid genotypes. Let the value of these be $J_{11}, J_{12}, J_{21}$, and $J_{22}$ where, for example, $J_{12}$ is the value of the flux for the wild type at locus $j$ and the mutant at locus $k$. This is

$$
J_{12}=\left(X_{\mathrm{A}}-X_{\mathrm{B}} / K_{\mathrm{A}, \mathrm{B}}\right) /\left(1 / E_{1_{\hat{j}}}+1 / E_{2 k}+K\right),
$$

where $K$ is the sum of reciprocals of enzyme activities other than $j$ and $k$ as in (4). Similar expressions can, of course, be written down for the other three genotypes. An explicit expression for the additive-by-additive interaction variance, $V_{1}$ can be obtained using Kojima's (1959) method of differentiating the mean genotype value with respect to the gene frequencies, $q_{j}$ and $q_{k}$. This is

$$
\begin{aligned}
V_{\mathrm{I}} & =q_{j}\left(1-q_{j}\right) q_{k}\left(1-q_{k}\right)\left[\partial^{2} m /\left(\partial q_{j} \partial q_{k}\right)\right]^{2} \\
& =q_{j}\left(1-q_{j}\right) q_{k}\left(1-q_{k}\right) \epsilon^{2},
\end{aligned}
$$

where $\epsilon=J_{11}-J_{12}-J_{21}+J_{22}$, the interaction term for the two-locus case. Consider the ratio of the additive to interaction variance, $V_{\mathrm{A}} / V_{\mathrm{I}}$. From (8) and (12) and after rearrangement, this is

$$
\begin{aligned}
V_{\mathrm{A}} / V_{\mathrm{I}}=\left(a_{1} / \epsilon+\right. & \left.q_{\mathrm{k}}-1\right)^{2} /\left[q_{k}\left(1-q_{k}\right)\right] \\
& +\left(\mathrm{a}_{2} / \epsilon+\mathrm{q}_{j}-1\right)^{2} /\left[\mathrm{q}_{j}\left(1-\mathrm{q}_{j}\right)\right]
\end{aligned}
$$

where $a_{1}=J_{11}-J_{21}$ and similarly $a_{2}=J_{11}-J_{12}$. The ratio of variances as a function of gene frequencies and enzyme activities can be obtained by substituting expressions of type (11) for the flux into (13). This has the following properties.

1. $V_{A} / V_{\mathrm{I}}$ is independent of the environmentally controlled parameter $X_{\mathrm{A}}-X_{\mathrm{B}} / K_{\mathrm{A}, \mathrm{B}}$.

2. The values of the terms $a_{1} / \epsilon$ and $a_{2} / \epsilon$ are in the range one to infinity because both have the form $x /(x$ $-y), 0<y<x$. A very large value of either $a_{1} / \epsilon$ of $a_{2} /$ $\epsilon$ implies very little interaction variance relative to additive variance for all $q_{j}$ and $q_{k}$. It can easily be shown that this occurs as $E_{2 j} / E_{1 j} \rightarrow 1$ and/or $E_{2 k} / E_{1 k}$ $\rightarrow 1$. The interaction variance is highest relative to the additive variance as $a_{1} / \epsilon \rightarrow 1$ and $a_{2} / \epsilon \rightarrow 1$, i.e. when $J_{22}-J_{12}$ and $J_{22}-J_{21}$ become small. This occurs as the activities of the mutant alleles approach zero (null alleles).

3. With a small interaction term $(\epsilon \rightarrow 0), V_{\mathrm{A}} / V_{\mathrm{I}}$ is at a maximum as a function of gene frequency when $q_{j}$ and $q_{k}$ are $1 / 2$.

4. With null alleles at both loci [which gives maximum epistasis, see (2) above], the interaction variance is highest relative to the additive variance as $q_{j} \rightarrow 0$ and $q_{k} \rightarrow 0$ (i.e., the mutant alleles are common).

5. The activities of the nonvariable enzymes in the pathway are represented in the constant $K$. As $K \rightarrow \infty$ and if the mutant activities are not null, $V_{\mathrm{A}} / V_{\mathrm{I}}$ becomes very large because $a_{1} / \epsilon \rightarrow \infty$ and $a_{2} / \epsilon \rightarrow \infty$. This implies that the interaction variance is small relative to the additive variance if most of the control of flux lies with nonvariable loci (KACSER and BURNS 1973, 1981).

Multiple locus interactions: Interactions between pairs of loci are of lowest order, but multiple locus interactions also contribute to the genetic variance. Using KoJIMA's (1959) method, it is possible to obtain expressions for the variance contributed by higher order interactions, but these are very complex and the general properties are better illustrated by evaluating particular parameter sets on a computer. Figure 3 shows results for a linear chain of ten equivalent loci for a range of values of the ratio of mutant to wild-type activity, $E_{2} / E_{1}$. Results for three gene frequencies of the wild type are shown $(q=0.1$, mutant allele common; $q=0.5 ; q=0.9$, mutant allele rare), and are expressed as the proportion of interaction variance, $V_{\mathrm{l}} / V_{\mathrm{T}}$. Each step on the horizontal axis represents changing one nonvariable locus in the chain to a variable locus of given gene frequency in the population. 

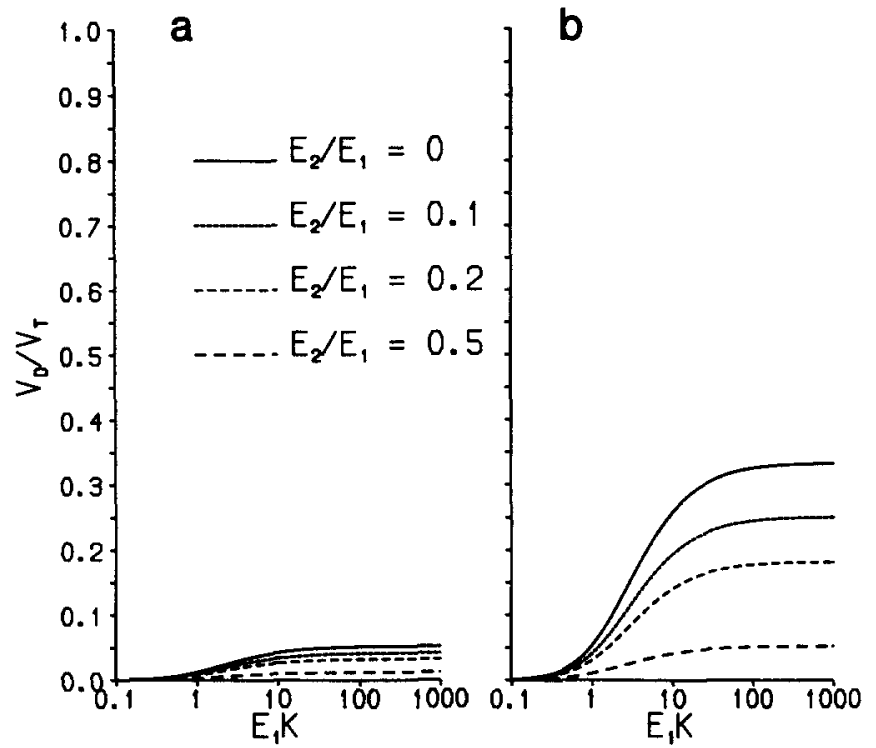

As indicated by (13), with two variable loci only, the proportion of interaction variance is highest for null mutant alleles when the mutant allele is common $(q=0.1$ in Figure 3$)$. The presence of further variable loci does not necessarily lead to an increase in the proportion of interaction variance. Why is this so? Although an increase in the number of variable loci leads to an increase in the number of interaction terms, increasing the number of mutant alleles segregating lowers the average activities of the enzymes in the chain. This has the consequence that the average change in flux between mutant and wild type becomes more linear, hence the presence of maxima for all cases except the special case of null mutant activity in Figure $3 \mathrm{a}$ where mutant alleles are at high frequency. The linear increase in the proportion of interaction variance in Figure $3 c$ is simply a consequence of a linear increase in the ratio of the number of two locus interaction terms to number of additive terms; higher order interactions contribute little variance because genotypes with more than two mutant alleles are very rare.

\section{Branched pathway}

Variation in the reference branch: For the case of two variable loci in the reference branch, it can be shown from (6) that the flux in the reference branch has the same form as (11) except that the term $K$ is replaced with,

$$
K=b^{\prime}+1 /\left(1 / a K_{\mathrm{A}, \mathrm{S}}+1 / c\right),
$$

where $b^{\prime}$ is the sum of reciprocals of enzyme activities in the reference branch excluding the activities of the two variable loci as in (4). Thus, the properties described for the linear chain are the same as described for this case. As $K \rightarrow \infty$, the control of the reference flux shifts to the enzymes in the nonvariable segments of the system, and the proportion of interaction variance decreases.

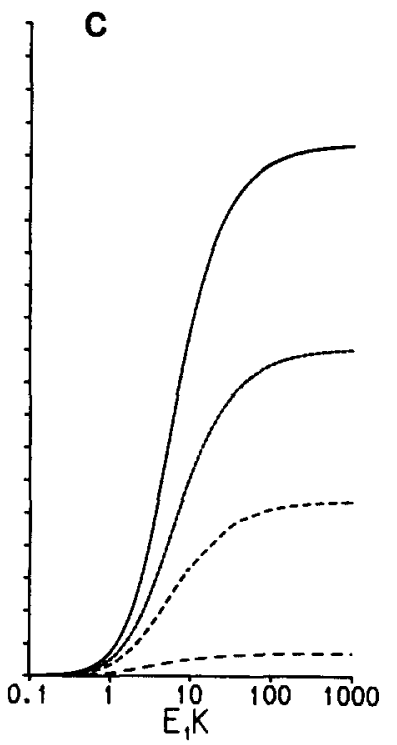

FiguRE 6.-The proportion of dominance variance of flux in a linear chain (Figure 1). The horizontal axis is the product of wild-type activity at the segregating locus and the sum of reciprocals of activities of the rest of the loci in the chain. Each curve relates the variance component for different values of mutant to wild type activity ratio, $E_{2} / E_{1}$. (a) $q=0.1$; (b) $q$ $=0.5 ;$ (c) $q=0.9$.
With more than two varying loci in the reference branch, the interaction component changes with increasing number of segregating loci as in Figure 3 (results not shown), i.e., $V_{1} / V_{\mathrm{T}}$ can have a maximum greater than that obtained when all loci are segregating.

Two-locus interactions between loci in different branches: With two segregating loci, at least one of which is either in the common or competing branches, the behavior is rather different from the case of both loci in the reference branch. A change in activity of a locus in either the common or competing branches can lead in theory to a reversal in the direction of fluxes [see KACSER (1983) for an analysis of the behavior of branched pathways in terms of control analysis]. The values of $a_{1} / \epsilon$ and $a_{2} / \epsilon$ in (13) are no longer constrained by $1<a_{1} / \epsilon, a_{2} / \epsilon<\infty$. The ratio of additive to interaction variances is a much more complex function and involves the concentrations of the externally controlled substances as well as equilibrium constants which are involved in determining the reversibility of the fluxes. This is illustrated in Figure 4 which shows (a) the value of $a_{1} / \epsilon$, and (b) the response of the reference flux as $X_{\mathrm{B}}$ is modulated. The activities of the wild type alleles at the two loci are equivalent and the mutant alleles are both nulls.

As the value of the flux for the double wild type, $J_{11}$, approaches zero, the value of $a_{1} / \epsilon$ also approaches zero. Near this point, a change in direction of the flux occurs, and (13) indicates that the proportion of interaction variance can be substantial as $q_{k} \rightarrow 1$ (mutant allele rare), which contrasts with the result for variation in the reference branch only. KACSER (1983) showed that a change in direction of the flux can occur for a change in enzyme activity with a high control coefficient. The dependence of the proportion of interaction variance on the $X$ 's in the branched system contrasts with its independence in the linear pathway of $X_{\mathrm{A}}$ and $X_{\mathrm{B}}$. 
Multiple-locus interactions in the branched system: The effect on the interaction component of variance of many loci simultaneously segregating in each of the three branches is illustrated in Figure 5. In this system, the thermodynamic pressures (i.e., concentration difference in relation to equilibrium constant) for the reactions $\mathrm{X}_{\mathrm{A}} \rightarrow \mathrm{X}_{\mathrm{B}}$ and $\mathrm{X}_{\mathrm{A}} \rightarrow \mathrm{X}_{\mathrm{C}}$ are large relative to the thermodynamic pressure between the outputs. The figure compares the effects of equivalent loci in a "symmetrical" system, $X_{\mathrm{B}}=X_{\mathrm{C}}$ and in two "asymmetrical" systems where $X_{\mathrm{B}} \gg X_{\mathrm{C}}$ or $X_{\mathrm{C}}$ $\gg X_{\mathrm{B}}$, and can be compared to the interaction variance generated by a linear pathway (Figure 3 ). The graphs have uneven shapes because the segregating loci are introduced into the three branches successively. Figure 5 suggests that the effect of asymmetry is rather small because the three types of system give similar properties of interaction variance. Most of the interaction in this case occurs between loci in the common ("A") branch and the reference ("B") branches because most of the increase in interaction occurs with introduction of segregating loci in these branches; the introduction of segregating loci in the competing branch does not lead to substantial increases in the proportion of interaction variance.

\section{Dominance variance}

We have been concerned primarily with the interaction component of nonadditive variance, but in a diploid population a dominance component is present because of interactions between alleles within loci. The analysis of the interaction component also becomes more complicated because of the presence of additive by dominance and dominance by dominance interactions. These are higher order than additive by additive or dominance interactions, they are functions of third and higher derivatives of the population mean with respect to gene frequency and contain an additional $q(1-q)$ term for each derivative (KojIMA 1959). They are therefore likely to be small. It is worthwhile exploring the consequences of dominance in metabolic pathways due to two alleles segregating at one locus, and to evaluate the dominance component of variance, thus extending the treatment of KACSER and Burns (1981).

Assume that the activity of the enzyme in the heterozygote is the average of the two homozygotes, which holds for most cases (KACSER and BURNS 1981). Using similar terminology to the haploid case, let the fluxes of the homozygous wild type, homozygous mutant, and heterozygote be $J_{1}=C_{\mathrm{x}} /\left(1 / E_{1}+K\right), J_{2}=$ $C_{\mathrm{X}} /\left(1 / E_{2}+K\right)$, and $J_{3}=C_{\mathrm{X}} /\left(2 /\left(E_{1}+E_{2}\right)+K\right)$ respectively, where $C_{\mathrm{X}}=X_{\mathrm{A}}-X_{\mathrm{B}} / K_{\mathrm{A}, \mathrm{B}}$ and $K$ is the sum of reciprocals of the nonvariable loci as in (4). With Hardy-Weinberg equilibrium, the population mean is

$$
m=q^{2} J_{1}+2 q(1-q) J_{3}+(1-q)^{2} J_{2} .
$$

Expressions for the additive and dominance variances can be obtained by KoJIMA's (1959) method. The ratio of additive to dominance variance is,

$$
\begin{aligned}
V_{\mathrm{A}} / V_{\mathrm{D}}= & 2\left[q(1-q)(\partial m / \partial q)^{2}\right] / \\
& {\left[q^{2}(1-q)^{2}\left(\partial^{2} m / \partial q^{2}\right)^{2}\right] } \\
= & 2\left[q+\left(J_{3}-J_{2}\right) /\left(J_{1}-2 J_{3}+J_{2}\right)\right]^{2} / \\
& {[q(1-q)] . }
\end{aligned}
$$

Substituting expressions for the fluxes of the three genotypes for the case of the linear chain gives,

$$
\begin{aligned}
& V_{\mathrm{A}} / V_{\mathrm{D}}=2\left[q-\left(1+1 /\left(E_{1} K\right)\right) /\right. \\
&\left.\left(1-E_{2} / E_{1}\right)\right]^{2} /[q(1-q)] .
\end{aligned}
$$

Thus, the ratio of variances is a function of gene frequency, $q, E_{1} K$ and $E_{2} / E_{1}$, and has the following properties.

1. As the ratio of mutant to wild type activity approaches unity $\left(E_{2} / E_{1} \rightarrow 1\right)$, the variance becomes mainly additive.

2. As $E_{1} K \rightarrow 0$ the variance becomes mainly additive. With the nonvariable enzymes condensed to a single step, $E_{1} K$ can be rewritten as $E_{1} / E_{\mathrm{G}}$ with $E_{\mathrm{G}}$ the "group enzyme activity" (KeIGHTLEY and KACSER 1987). As $E_{1} / E_{\mathrm{G}} \rightarrow$ 0 , most of the control of the flux shifts to the variable locus implying a linear change of flux with change of $E_{1}$. The dominance deviation is therefore small.

3 . If the heterozygote flux is nearly intermediate, the dominance variance is greatest relative to the additive at intermediate gene frequency $(q \rightarrow 1 / 2)$.

4. As $E_{2} / E_{1} \rightarrow 0$, the dominance variance is greatest relative to the additive when the mutant allele is rare $(q \rightarrow 1)$. Note, this is the opposite of the condition for maximum additive by additive interaction variance in the haploid case.

These properties are illustrated in Figure 6 which shows the proportion of dominance variance of flux, $V_{\mathrm{D}} / V_{\mathrm{T}}$, for three gene frequencies of the wild-type allele and a range of values of $E_{2} / E_{1}$. For example, with five equivalent enzymes in the pathway $\left(E_{1} K=\right.$ $4)$ and the mutant allele at frequency $0.1(q=0.9)$, the proportion of dominance variance is almost $26 \%$ for a null mutant allele, but only $2 \%$ for $E_{2} / E_{1}=0.5$.

\section{DISCUSSION}

Nonadditive variance of flux: Allelic variation of enzyme activity generates both interaction and dominance variance of metabolic flux, but these are present in different circumstances. When the ratio of mutant to wild-type activity is small, the proportion of interaction variance is maximal as the mutant alleles become common. The proportion of dominance variance in a diploid population is at a maximum as the mutant allele becomes rare. With a small difference between mutant and wild type, both nonadditive components are maximal at intermediate gene frequencies. 
There are implications of the above for responses to directional selection of metabolic flux. Assume that there is a range of allelic effects segregating at different loci controlling the flux in a pathway. Selection to increase the flux in the pathway will tend to fix the alleles of largest effect quickest. Their fixation leads to a reduction in their control of the flux because there is an inverse relationship between control of flux and activity at a locus. The loci at which alleles of smaller effect segregate must as a consequence increase their share of flux control, so the effect of allelic variation at these loci becomes larger (KACSER and Burns 1981). This is similar to the epistatic effect described by DykHuizen, Dean and Hartl (1987). Thus the epistasis allows new variation to be revealed as upward selection progresses, but a reverse attenuation effect occurs for selection of flux in the downward direction. In a haploid population, an asymmetrical response pattern is therefore expected. For a diploid population, directional dominance of alleles controlling flux is present. This causes an asymmetry in the opposite direction because the effects of mutant alleles are hidden in heterozygotes, so responses therefore show less asymmetry (P. D. KeightLeY and H. KACSER, unpublished data). The presence of nonsegregating loci also affects dominance and additive by additive variances in different ways. As control of flux shifts to nonvariable loci $(K \rightarrow \infty)$, the proportion of interaction variance becomes small, but the proportion of dominance variance can become large as $E_{2} /$ $E_{1} \rightarrow 0$.

Where null alleles contribute to enzyme activity variation, the interaction component increases monotonically with increasing number of varying loci. With mutant alleles of other than null activity, increasing the amount of allelic variation can actually decrease the proportion of interaction variance. This somewhat surprising result can be understood in terms of the distribution of flux control coefficients in the pathway. The introduction of further mutant alleles, while producing an increase in the number of interactions between loci, leads on average to a more linear change in flux from allelic substitution at any one locus. This linear change is less affected by allelic variation in enzyme activity at other loci.

The behavior of the linear chain also applies to the branched system for alleles affecting enzyme activities within the branch in which the flux is measured (reference branch). The interaction component depends on the activity differences at the loci contributing to the flux variation, and also on the activities of the enzymes elsewhere in the system including the other branches, and decreases as their controlling influence on the flux increases.

The case of enzyme activity variation in different branches is more complex. In a branched pathway, changes in enzyme activity can, in theory, cause a change in the direction of flow, but since this situation is rather unlikely in nature, cases of large thermodynamic pressure from input $\left(X_{A}\right)$ to outputs $\left(X_{B}\right.$ and $\mathrm{X}_{\mathrm{C}}$ ) are most relevant. In the branched pathway, the interaction component depends on the "externally determined" equilibrium constants and on the source $\left(X_{\mathrm{A}}\right)$ and sink $\left(X_{\mathrm{B}}\right.$ and $\left.X_{\mathrm{C}}\right)$ concentrations. It is therefore more difficult to generalize about epistasis in such a system. However, when the thermodynamic pressure from inputs to outputs is high relative to the pressure between the outputs, most of the interaction occurs between loci in the reference and the common branch. Presumably such loci contribute most substantially to the total variance, the loci in the competing branch being more kinetically distant (KACSER 1983). A possible exception can occur when the reference flux is a small "leak" relative to the competing flux. In this case, variation in the common and competing branches is likely to contribute substantially to the total genotypic variance in the reference flux and also to the interaction component should allelic activity differences be large. This "branch point effect" has been investigated previously by KACSER (1983) and by LAPORTE, WALSH and KOSHLAND (1984).

In vitro enzyme activity differences: In this analysis, the specific activity of an enzyme is assumed to be proportional to the product of its turnover number and concentration. As well as changes in the structure of the enzyme molecule (which can affect turnover number and stability and hence its concentration), there are many classes of mutation capable of affecting both parameters. Such mutations include gene duplications and deletions, mutations at promoters, those affecting the stability and translation rate of mRNA, and mutations affecting the concentration and activity of "effector" molecules. Thus, enzyme activity is itself a polygenic character for which there is evidence from studies of enzyme activity variation in natural populations of Drosophila (LAURIE-AHLBERG et al. 1980; LaUrie-Ahlberg 1982; Graf and Ayala 1986), and in mice (PAIGEN 1979). Data on enzyme activity variation in natural and artificial populations fall into four main classes: (1) in vitro determination of activities of allozymes; (2) in vitro activity differences between Drosophila chromosome substitution lines (e.g., LAURIE-AHLBERG et al. 1980; LAURIE-AHLBERG, 1982; Graf and Ayala 1986; MiYashita and LaURIE-AhLBERG 1986); (3) in vitro enzyme activity differences between inbred strains of mice. The measured enzyme activities vary widely between lines and the differences frequently depend on the tissue in which the activity is measured (e.g., Bulfield, MOORE and KACSER 1978; JoHNSON and Hong 1986; JoHnson, Hong and KNIGHTS 1986); and (4) in vitro activity differences between artificially selected lines. Various enzyme activities thought likely to contribute to characters of economic importance in a number of species have 
been measured, including chickens (BANNISTER et al. 1984; WhITEHEAD et al. 1984; ASANTE and Bulfield 1988), mice (ASANTE 1988), and pigs (STANDAL and VANGEN 1980). In the above studies, the largest range of enzyme activities has been detected between mouse inbred strains. In these and other studies, however, small and nonsignificant differences in activity were most common (i.e., less than a factor of four), with an increasingly small proportion of more extreme activity differences. The large activity differences measured between inbred mouse lines may reflect their polyphyletic origins (BonHOMME et al. 1987).

It is not possible to relate these observations directly to the biochemical models investigated here. These biochemical models are very simple approximations of in vivo pathways, a consequence of the need to incorporate a genetic model of enzyme activity variation segregating in a population. However, the models show that small changes in enzyme activity (as observed in the in vitro studies) generate very little additive by additive interaction and dominance variance of flux as a proportion of the total genetic variance. Mutant alleles of low activity and at low frequencies contribute mainly dominance variance, but little interaction variance. This prediction of lack of interaction contrasts with the highly interactive nature of metabolic pathways within which enzymes are embedded. Conversely, the observation of substantial additivity for variation in a character does not necessarily imply that the components contributing to the variation are acting independently.

1 wish to thank H. KACSER and W. G. HILl for helpful discussions and N. Wray for commenting on the manuscript. This work was supported by a grant from the Agricultural and Food Research Council.

\section{LITERATURE CITED}

Asante, E. A., 1988 Biochemical genetics of lipid metabolism in chickens and mice. Ph.D. thesis, Edinburgh University.

Asante, E. A., and G. Bulfield, 1988 Activities of NADPHgenerating enzymes in genetically fat and lean chickens, in Leanness in Domestic Birds, edited by B. LECLERCQ and C. C. WHITEHEAD. Butterworths, London.

Bannister, D. W., A. Lee, C. Whitehead and H. Griffin, 1984 Lipogenic enzyme activity and fructose 2,6-bisphosphate concentrations in livers of two lines of domestic fowl (Gallus domesticus) selected for different body fat content. Int. J. Biochem. 16: 1301-1305.

Bonhomme, F., J-L. Guenet, B. Dod, K. MoRiwaki and G. BulFIELD, 1987 The polyphyletic origin of laboratory inbred mice and their rate of evolution. Biol. J. Linn. Soc. 30: 51-58.

Bulfiel.d, G., E. A. MOORE and H. KACSER, 1978 Genetic variation in activity of the enzymes of glycolysis and gluconeogenesis between inbred strains of mice. Genetics 89: 551-561.

Cleland, W. W., 1963 The kinetics of enzyme catalysed reactions with two or more substrates or products. I. Nomenclature and rate equations. Biochim. Biophys. Acta 67: 104-137.

Dykhuizen, D. E., A. M. Dean and D. L. Har'TL, 1987 Metabolic flux and fitness. Genetics 115: 25-31.

FALCONER, D. S., 1981 Introduction to Quantitative Genetics,Ed. 2. Longman, London.

Graf, J-D., and F. J. Ayala, 1986 Genetic variation for superoxide dismutase level in Drosophila melanogaster. Biochem. Genet. 24: 153-168.

Johnson, W. G., and J. L. HoNG, 1986 Variation in $\alpha$-L-fucosidase properties among 28 inbred mouse strains: six strains have high enzyme activity and heat-stabile enzyme with a variant $\mathrm{pH}$ activity curve; twenty-two strains have low activity and heatlabile enzyme. Biochem. Genet. 24: 469-483.

Johnson, W. G., J. L. HonG and S. M. KNights, 1986 Variation in ten lysosomal hydrolase enzyme activities in inbred mouse strains. Biochem. Genet. 24: 891-909.

KACSER, H., 1983 The control of enzyme systems in vivo: elasticity analysis of the steady state. Biochem. Soc. Trans. 11: 35-40.

Kacser, H., and J. A. Burns, 1973 The control of flux. Symp. Soc. Exp. Biol. 27: 65-104.

KaCSER, H., and J. A. Burns, 1981 The molecular basis of dominance. Genetics 97: 639-666.

KaCSer, H., and J. W. Porteous, 1987 Control of metabolism: what do we have to measure? Trends Biochem. Sci. 12: 5-14.

KeightLeY, P. D., and H. KACSER, 1987 Dominance, pleiotropy and metabolic structure. Genetics 117: 319-329.

KoJIMA, K., 1959 Role of epistasis and overdominance in stability of equilibria with selection. Proc. Natl. Acad. Sci. USA 45: 984-989.

Laporte, D. C., K. Walsh and D. E. Koshland, 1984 The branch point effect. J. Biol. Chem. 259: 14068-14075.

LAURIE-Ahl.BERG, C. C., 1982 Genetic, ontogenetic and tissuespecific variation of dipeptidases in Drosophila melanogaster. Biochem. Genet. 20: 407-424.

Laurie-Ahlberg, C. C., G. Maroni, G. C. Bewley, J. C. Lucchesi and B. S. WEIR, 1980 Quantitative genetic variation of enzyme activities in natural populations of Drosophila melanogaster. Proc. Natl. Acad. Sci. USA 77: 1073-1077.

Miyashita, N., and C. C. LAurie-Ahlberg, 1986 Developmental variation in effects of the second and third chromosomes on the activities of the glucose 6-phosphate and 6-phosphogluconate dehydrogenases in Drosophila melanogaster. Biochem. Genet. 24: 447-467.

Paigen, K., 1979 Acid hydrolases as models of genetic control. Annu. Rev. Genet. 13: 417-466.

SAvageaU, M. A., 1969 Biochemical systems analysis. I. Some mathematical properties of the rate law for the component enzymatic reactions. J. Theor. Biol. 25: 365-369.

SavageaU, M. A., E. O. VoIT and D. H. IRvine, 1987 Biochemical systems theory and metabolic control theory. 1. Fundamental similarities and differences. Math. Biosci. 86: 127-148.

Standal, N., and O. VANGen, 1980 Physiological effects of selection for growth rate and backfat thickness, pp. 125-130 in Selection Experiments in Laboratory and Domestic Animals, edited by A. RoBertson. Commonwealth Agricultural Bureaux, Slough.

Whitehead, C. C., R. L. Hood, G. S. Heard and R. A. E. PyM, 1984 Comparison of plasma very low density lipoproteins and lipogenic enzymes as predictors of fat content and food conversion efficiency in selected lines of broiler chickens. $\mathrm{Br}$. Poult. Sci. 25: 277-286.

Communicating editor: M. TURELLI 